\title{
Promising alternative settings for HPV vaccination of US adolescents
}

\author{
Parth D. Shah ${ }^{1}$, Melissa B. Gilkey ${ }^{2}$, Jessica K. Pepper ${ }^{1,2}$, Sami L. Gottlieb ${ }^{3}$, and Noel T. \\ Brewer $^{*}, 1,2$ \\ ${ }^{1}$ Department of Health Behavior, UNC Gillings School of Global Public Health, Chapel Hill, NC \\ 27599-7440, USA 2UNC Lineberger Comprehensive Cancer Center, Chapel Hill, NC, USA \\ ${ }^{3}$ Medical Epidemiologist, Chavannes-de-Bogis, Switzerland
}

\begin{abstract}
Vaccination in alternative settings, defined here as being outside of traditional primary care, can help address the pressing public health problem of low human papillomavirus vaccine coverage among adolescents in the United States. Pharmacies are promising because they are highly accessible and have well established immunization practices. However, pharmacies currently face policy and reimbursement challenges. School-located mass vaccination programs are also promising because of their high reach and demonstrated success in providing other vaccines, but control by local policymakers and challenges in establishing community partnerships complicate widespread implementation. Health centers in schools are currently too few to greatly increase access to human papillomavirus vaccine. Specialty clinics have experience with vaccination, but the older age of their patient populations limits their reach. Future steps to making alternative settings a success include expanding their use of statewide vaccine registries and improving their coordination with primary care providers.
\end{abstract}

\section{Keywords}

adolescent vaccines; alternative vaccination settings; human papillomavirus; pharmacists; school vaccination

Widespread vaccination against HPV could prevent more than two-thirds of cervical cancer cases [1] and reduce the incidence of vulvar, vaginal, penile, anal and possibly

\footnotetext{
(C) 2014 Informa UK Ltd

*Author for correspondence: Tel.: +1 919966 3282, Fax: +1 919966 2921, ntb@unc.edu.

Financial \& competing interests disclosure

PD Shah and MB Gilkey drafted the manuscript. PD Shah, MB Gilkey, JK Pepper, SL Gottlieb and NT Brewer critically reviewed and revised the manuscript. The authors received no honorarium, grant or other form of payment to produce the manuscript. The secondary analyses of publicly available data reported in this manuscript did not require institutional review board approval. NT Brewer received grants and/or honoraria from Merk Sharp \& Dohme and from GlaxoSmithKline, unrelated to this manuscript. MB Gilkey received funding from the Cancer Control Education Program at UNC Line-berger Comprehensive Cancer Center (R25 CA57726). The authors have no relevant affiliations or financial involvement with any organization or entity with a financial interest in or financial conflict with the subject matter or materials discussed in the manuscript apart from those disclosed.

The authors did not receive any writing assistance in the production of this manuscript.
} 
oropharyngeal cancers, as well as genital warts [2]. As cervical cancer and other HPVrelated cancers disproportionately affect minority, rural and low-income individuals, HPV vaccine is particularly important for adolescents in these populations [3]. National guidelines recommend routinely providing HPV vaccine to adolescents aged 11-12, with catch-up doses for males aged 13-21 and females aged 13-26 [4,5]. For this reason, our article focuses on adolescents aged 11-12. Although these guidelines took effect in 2007 for females and 2011 for males, HPV vaccination coverage is low and has improved little in recent years. By 2012, 33\% of girls and 7\% of boys aged 13-17 had received the recommended three doses [6]. Rates for adolescent girls are largely unchanged from the previous year. HPV vaccine coverage is far lower among age-eligible adolescents than coverage for other recommended adolescent vaccines, including the combination tetanus, diphtheria and pertussis (Tdap) vaccine ( $85 \%$ coverage) and meningococcal conjugate vaccine (74\% coverage) [6].

Solutions to low vaccine coverage include encouraging healthcare providers to routinely offer strong recommendations for HPV vaccine and increasing parent awareness that the vaccine is for both adolescent boys and girls. While pediatric and family medicine clinics are the primary settings for HPV vaccine administration [7], adolescents visit these settings less often than younger children. Most adolescent attend at least one healthcare visit a year, but many of these visits are for acute care when vaccination is rarely discussed. Nearly half of US children aged 12-17 do not have reliable access to comprehensive primary care [8]. Too few primary care providers serve rural and disadvantaged urban areas [9], one reason that adolescents from vulnerable groups are less likely to obtain consistent primary care $[10,11]$. The need for multiple visits to complete the HPV vaccine series amplifies these problems. Expanding vaccination services to locations outside of traditional settings is one way to increase HPV vaccination rates, especially for adolescents with limited access to primary care. The US President's Cancer Panel endorsed alternative vaccination settings as a promising way to increase HPV vaccine coverage in the USA [12]. These alternative settings can support and fill in gaps in access to primary care.

A CDC working group reviewed several candidate settings outside of pediatrics and family medicine for adolescent vaccine administration, including pharmacies, health departments, family planning clinics and emergency departments [13]. While the working group concluded that these settings were safe and appropriate to provide immunizations to adolescents, the group completed their work over 5 years ago, did not consider HPV vaccine specifically and did not review potentially relevant settings (e.g., school health centers, mass vaccinations programs in schools and dental offices). The aim of this article is to review traditional and alternative settings in light of the unique challenges posed by HPV vaccination in the USA with particular attention to three considerations: reach or providers' ability to access adolescents aged 11-12, with special emphasis on populations at higher-risk for HPV-related cancers later in life; acceptability and convenience of alternative settings for adolescents and their parents, healthcare providers and other stakeholders; and feasibility of vaccine delivery in terms of storage, availability of healthcare providers trained in vaccination, documentation of and remuneration for vaccine doses and policy environment. Table 1 summarizes the general findings for each setting. 


\section{Traditional settings}

\section{Primary care in pediatric \& family medicine clinics}

Reach-Primary care clinics and practices are the most common setting for prescribing adolescent vaccines, usually during visits with pediatricians and family medicine physicians [7,14]. Most primary care clinics provide good access to adolescents aged 11-12, though access to some populations at higher-risk for HPV-related cancers may be lower [10]. One challenge is that, compared with young children, adolescents, particularly boys, make fewer preventive care visits in general $[14,15]$, reducing opportunities to provide vaccines and increasing patient loss to follow-up. This challenge is exacerbated by most adolescents' healthcare visits being for acute rather than preventive care. Even though adolescents' less frequent contact with pediatric and family medicine clinics may affect immunization coverage for all vaccines, HPV vaccination is likely disproportionately impacted given the number of visits needed to complete the three-dose series.

Acceptability-Another strength of the primary care setting is that parents overwhelmingly indicate that a physician's clinic is the most desirable setting for HPV vaccination, for reasons including familiarity with and trust in their adolescents' providers and ease of tracking vaccinations [16,17]. A barrier to HPV vaccination in primary care setting is inconvenience to parents. Additional visits required for series completion compound the problems of scheduling visits with a clinic that may offer limited hours, the need to travel to a location that may not be close and time spent in the waiting room [18]. Some medical practices offer 'vaccination only' visits with short wait times to see a nurse, but the prevalence of this practice is not well documented.

Healthcare provider recommendation is by far the strongest predictor of HPV vaccine uptake [19-25]. However, primary care providers miss most opportunities to recommend the vaccine to eligible adolescent patients. Lack of a provider recommendation is one of the most common reasons parents of unvaccinated children give for not getting HPV vaccine. Furthermore, qualitative research suggests that recommendations that providers do deliver for HPV vaccine are often weaker than for other vaccines [26-28]. Although reasons are complex, providers' current practices may be due to discomfort talking about sex with adolescents and parents [29].

Another challenge may be physician unfamiliarity or disagreement with the Advisory Committee on Immunization Practices (ACIP) guidelines. For instance, primary care providers are less likely to recommend HPV vaccine to younger adolescents than older ones, despite ACIP recommendations to target patients aged 11-12 [30,31]. Providers may not view younger adolescents as being at risk for contracting HPV, and therefore, they may see no harm in delaying vaccination [31]. Addressing missed opportunities is an especially promising way to increase HPV vaccination coverage.

Feasibility-As primary care settings have a long-standing history of vaccination, the feasibility of HPV vaccination in these settings is generally very high. Primary care clinics have the personnel and infrastructure to store and deliver vaccine doses safely and effectively, and they also typically have insurance billing systems. This primary care- 
centered approach has been successful for Tdap and meningococcal vaccines, demonstrating that it is possible to reach the vast majority of adolescents through primary care clinics [28]. The CDC estimates that if providers gave HPV vaccine concomitantly with other vaccines during an adolescent girl's health visit, HPV series initiation would be above $90 \%$ with lower series completion [28]. Even though most adolescents have a medical home and make at least one visit yearly [32], providers still miss many opportunities to provide HPV vaccine concomitantly with other adolescent vaccines $[28,33]$.

\section{Health department immunization clinics}

Reach-Health department immunization clinics are vital safety-net providers of immunizations to those who cannot pay or do not have access to a primary care provider [13]. Health departments provide the most HPV vaccinations outside of pediatric and family medicine clinics, but the absolute number is relatively low [7]. Compared with primary care clinics, health departments serve many fewer adolescents, but the patients they serve may be more likely to be from populations at higher risk for HPV-related cancers (e.g., minority, low income). Furthermore, health departments likely play an essential role for vaccination referrals, as smaller or rural primary care clinics and practices may be less likely to stock adolescent vaccines, including HPV vaccine, due to cost of storage and inadequate reimbursement [34].

Acceptability-Adolescents and parents indicate that a health department immunization clinic is an acceptable alternative to a primary care clinic [16,35]. However, many barriers in a primary care setting related to convenience are likely also relevant to health departments.

Feasibility-Health department immunization clinics can store and distribute large quantities of vaccines [13], and most health departments already stock HPV vaccine [36]. However, health departments in different states show substantial variability in the amount of vaccine they store and who qualifies to receive it [36]. Some states provide universal coverage of vaccines, including HPV vaccine, while other states offer assistance only through the Vaccines for Children (VFC) program, which provides ACIP-recommended vaccines free of charge for adolescents who are uninsured, underinsured, Medicaid eligible, American Indian or Alaska Natives [36,37]. These policies for funding vaccine assistance programs affect most settings, but they may be especially pronounced in settings like health departments that serve primarily low-income clients. Private insurers may not reimburse for office visits and administration fees at health department immunization clinics; such fees could be a large barrier for low- and middle-income families.

\section{Promising alternative settings}

Pharmacies and schools are the two most promising alternative settings, largely because of the exceptional access they provide to adolescents and being acceptable to parents and adolescents, though these settings face meaningful challenges to implementing provision of HPV vaccine on a national scale. 


\section{Pharmacies}

Reach-An important advantage of pharmacies is that they have substantial access to adolescents. Consumers make an estimated 250 million visits to pharmacies each week [38]. In health professional shortage areas and rural areas, pharmacists are the most prevalent health-care providers [39] with around 275,000 licensed pharmacists practicing in around 70,000 pharmacies across the USA [40]. Given their high prevalence in disadvantaged areas, pharmacists may have the potential to reach pre-teens and adolescents who otherwise would not have regular access to preventive care [13,41]. For instance, a case study from Richmond, Virginia showed that one pharmacy chain successfully implemented an immunization program that vaccinated around 2500 children and adolescents, of whom 9095\% were uninsured [42].

Acceptability-Pharmacists are among the most trusted professionals in the USA, along with physicians and nurses [43]. The public supports pharmacist-provided immunizations for adults, and a growing literature shows that parents and adolescent children also support pharmacist provision of vaccines, including HPV vaccine [35,44]. Support is even stronger among parents who have adolescent children who have not had a recent visit to their healthcare provider $[35,44]$. In addition, pharmacies are open longer hours than most primary care clinics and generally require no appointments [41,45,46]. A study of California pharmacies found that adult vaccination at pharmacies was cheaper (lower cost per unit) and more likely to be consistent with ACIP guidelines than vaccination in primary care settings and mass immunization clinics [47]. It may follow that similar quality of care could be provided to adolescents in pharmacies. However, some primary care physicians are not yet comfortable with adolescent immunizations in pharmacies, arguing that the availability of stand-alone services will lead patients to have fewer primary care visits and receive less comprehensive preventive care [48]. To address these concerns, pharmacists who administer HPV vaccine must collaborate closely with primary care providers to ensure care coordination and build trust [48].

Feasibility-Pharmacists already provide HPV vaccine in the majority of the US states, but several challenges exist to more widespread implementation. Many colleges and schools of pharmacy place special emphasis on immunization training, enabling a new wave of pharmacists to join the workforce as trained immunizers [49]. Given over a decade of experience with providing adult immunizations, many pharmacies are equipped and staffed to extend such services to adolescents. Pharmacies are unique among the settings we reviewed in effectively generating demand for vaccination services through local and national advertising campaigns.

A feature of pharmacies that most other traditional and alternative settings lack is the ability to bill patients' insurance while they are at the pharmacy by performing immediate claims adjudication. While pharmacies typically do this for medications, they can also process claims for many adult vaccines (e.g., herpes zoster, pneumococcal and seasonal influenza). Thus, patients find out the cost of vaccination in real time. Although not yet widely tested, it may be feasible for pharmacies to conduct claims adjudication for adolescent vaccines. 
Success of these claims will depend on pharmacists achieving in-network status, which anecdotal evidence suggests is uncommon at present.

The largest structural drawback is that insurers often do not confer in-network status to pharmacists and pharmacies. Patients often have to pay out of pocket for pharmacydelivered vaccination or for administration costs even if they have private health insurance that covers the vaccine [41]. Costs for many adolescents receiving vaccines could be covered if pharmacists were VFC program providers. Pharmacist vaccination may be attractive to private insurers because it does not entail an office visit fee that is common in primary care settings.

Another barrier is that, although the number of states allowing adult vaccination at pharmacies increased dramatically from 9 in 1995 to all 50 in 2010 [49], many states restrict the vaccines pharmacies may offer as well as the age of patients that pharmacists may serve $[41,48]$. Currently, 45 states and US territories allow pharmacists to administer HPV vaccine, with 21 states allowing pharmacists to immunize adolescents at least 12 years of age with that vaccine [46]. However, the mechanisms by which pharmacists can immunize vary substantially by state (e.g., standing orders vs prescription), state laws may constrain pharmacists to vaccinating only females and some states do not permit pharmacists to vaccinate adolescents younger than age 18 [50]. While efforts to expand pharmacists' immunization authority are underway in several states, medical associations such as the American Association of Pediatrics have given only limited support to pharmacies as an alternative vaccination setting, endorsing pharmacist provision only if pediatric patients do not have reliable access to a primary care clinic [51].

\section{Schools}

\section{School health centers}

Reach: School health centers (SHCs) are clinics that provide students, and sometimes other family members, with comprehensive medical care, including primary care, mental health and nutrition counseling services [52]. The over 1900 SHCs that currently operate in the USA [52] are in 6\% of the nation's schools [53]. Although not yet widespread, the number of SHCs has grown over time, and these clinics have recently benefited from a 4-year, US \$200 million grant in federal funding from the Patient Protection and Affordable Care Act (ACA) of 2010 [54]. SHCs are more likely to serve schools with low-income, uninsured and minority students. However, many SHCs do not serve schools with adolescents, fewer than half of SHCs are located in rural areas, some SHCs are not located on school grounds and some states have few or no SHCs [52] limiting their overall reach.

Acceptability: Surveys suggest that parents view SHCs as one of the preferred alternative settings because of their convenience and familiarity [16]. Parents do not have to miss work to vaccinate their children since students are already attending school daily and using services at the health center [55]. Perhaps as a result, adolescents who receive HPV vaccine at SHCs are more likely to complete the three-dose series compared with patients vaccinated at community clinics [55]. 
Feasibility: Most SHCs (85\%) are able to provide vaccines [56,57]. In a 2007-2008 national survey of SHCs, $80 \%$ reported offering HPV vaccine [58]. However, a more recent North Carolina study found that while $60 \%$ of the state's SHCs had carried the vaccine the previous year, only around a quarter still carried it a year later, suggesting some challenges to feasibility or demand [59]. Strengths of SHCs include that they have primary care providers on staff an average of 31 hours per week [52]. In addition, SHCs are generally able to bill private and public third-party payers, and many offer subsidized vaccines through the VFC program and State Children's Health Insurance Plans [58]. Many SHCs are already using recommended strategies for tracking vaccinations, such as updating electronic health records and contacting primary care providers to inform them of students' immunizations [58]. Evidence shows that such tracking methods help increase immunization rates [60]. Challenges include that SHCs are highly heterogeneous with respect to administrative systems and policies, many have unstable or variable funding [61], and some require a separate vaccine consent in addition to general healthcare consent.

\section{Mass vaccination programs in schools}

Reach: Voluntary mass vaccination programs are partnerships between community providers (e.g., health departments) and schools, typically without health centers, to vaccinate students. These programs can reach most adolescents, since nearly all adolescents in the USA attend school [62]. Evidence shows that school vaccination programs have been successful both in the USA and abroad in providing vaccines to target populations. The UK and regions in Australia and Canada have achieved impressive HPV vaccine coverage of adolescent girls through voluntary school programs [63-67]. For example, a recent study in Canada found that school programs yielded $75 \% \mathrm{HPV}$ series completion rate while programs in medical clinics achieved only $37 \%$ [68]. In the USA, voluntary school-located programs have been highly successful in increasing coverage of hepatitis B and flu vaccines [69-71], though they have had more limited success for HPV vaccine [61]. While schools undoubtedly have the highest reach to adolescents eligible for HPV vaccine, creating mass vaccination programs in other non-health-related areas (e.g., churches, sporting events, retail outlets) might provide other opportunities to vaccinate boys and girls in the community [72].

Acceptability: Parents [35,73,74], adolescents [35,74] and physicians [56] generally support vaccinating at schools. Importantly, parents are more willing to vaccinate at schools if they have an adolescent who has not had a recent healthcare visit [44] or regular site for healthcare [75], is uninsured [44] or has been previously vaccinated at school [44]. Therefore, establishing targeted mass vaccination programs in school districts in which adolescents from underserved populations are disproportionately represented may have widespread support from the community. The American Association of Pediatrics has recommended increasing health providers' involvement with schools without health centers to help adolescent vaccine uptake [56]. Despite support for this approach from many parents and healthcare providers, some school leaders such as superintendents and principals may be less supportive [76], as they anticipate potential concerns from the many constituents to whom they answer. Including school leaders early in the planning process may lessen their concerns and improve support for program implementation [76]. 
Feasibility: Vaccination programs in schools have to compete with schools' main priorities (educating, transporting and feeding children) [56]. Superintendents and school boards must approve the program because local school boards each set their own policies, and the vaccination program must also obtain parental consent in advance of vaccinating children, as parents are not usually present. Mass vaccination campaigns are often conducted in partnership with organizations that can address several additional challenges, such as documenting vaccine doses given, reporting them to providers, billing insurance, training staff to administer vaccines and maintaining the necessary equipment and facilities [56]. Health departments are natural partners for these efforts as their personnel are trained to organize large groups of people for disaster and emergency management [77]. State and local budget cuts in the USA may reduce funding for school nurses who would then be less available to help with mass vaccination programs [78].

\section{Other alternative settings}

The remaining alternative settings have limited ability to systematically reach the nation's adolescents, variable acceptability, substantial challenges to feasibility or some combination of all three.

\section{Specialty practices}

\section{Obstetrics-gynecology practices}

Reach: Visits by young women to obstetrics-gynecology (OBGYNs) practices increase with age. By age 17, more adolescent females visit OBGYNs (33\% of seven million visits) than pediatricians (23\%), and by age 21 , over $60 \%$ of these visits are to OBGYNs [79]. However, these clinics do not see many girls in HPV vaccine's targeted age range (11-12 years), and they do not serve boys. As a result, the role of OBGYNs in HPV vaccination may be mostly limited to catch-up efforts among older adolescent girls and young women. However, OBGYNs may be able to use routine breast and cervical cancer screening visits with adolescents' mothers to recommend HPV vaccination for their daughters [80]. Women's experiences with cervical health (e.g., treatment for cervical dysplasia) are related to intentions to vaccinate their daughters against HPV in some studies [81].

Acceptability: A national survey of HPV vaccine-eligible females, aged 13-26, found that $89 \%$ would get the vaccine if their OBGYNs recommended it [82]. A recent study found that OBGYNs were more comfortable vaccinating older females than girls aged 11-12, perhaps because younger adolescents are a population these clinicians infrequently serve [83]. OBGYNs have been very supportive of HPV vaccination efforts, with strong endorsement by the American College of Obstetrics and Gynecology (ACOG) [84]. As ACOG recommendations may greatly influence OBGYNs' decisions to incorporate vaccines into their practices, the most recent ACOG endorsement may encourage even more of them to provide HPV vaccine.

Feasibility: Among OBGYN practices that administer any immunizations, HPV vaccine is the most commonly offered vaccine, and often the only one [85]. Over $90 \%$ of OBGYNs stock HPV vaccine [83]. However, only $27 \%$ of their eligible patients initiate the series, and 
less than a third of those who initiate go on to receive all three doses [83]. Despite the ability to stock and deliver the vaccine, OBGYNs report that barriers to HPV vaccination include financial concerns, such as the costs of ordering and storing the vaccine and inadequate reimbursement [83] and the belief that vaccines should be provided elsewhere [85-87].

\section{Sexually transmitted infection clinics}

Reach: Sexually transmitted infection (STI) clinics are unlikely to see the youngest adolescents who are the primary group for HPV vaccination. Males are twice as likely to seek care in an STI clinic as females [88]. Moreover, most adolescents who seek care in these clinics are already sexually active, increasing the likelihood of previous HPV infection. However, they are unlikely to have had all HPV strains that the vaccine protects against, and therefore still may benefit from receiving it. A positive however is that STI clinics serve a disproportionate number of adolescent from vulnerable US populations that are the hardest to reach including poor, uninsured and minority adolescents and young adults [13].

Acceptability: As the primary mission of publicly funded STI clinics is to prevent STI transmission and sequelae, their providers' and patients' interest in HPV vaccination may be high. However, few studies have evaluated parents' and adolescents' comfort with receiving HPV vaccine at STI clinics. Parents may not be as comfortable having their adolescents vaccinated in STI clinics given their already low preference for using family planning clinics [16] as an alternative setting.

Feasibility: STI clinics face barriers that may make them a less feasible setting for HPV vaccine delivery. Only 7 of the 42 STI clinics in the CDC STD Surveillance Network stock HPV vaccine, with one of the clinics restricting its administration to females [89]. Clinics that wish to administer HPV vaccine in their immunization services may come across similar challenges previously seen in hepatitis B vaccinations [89] including cost of the vaccine, lack of resources related to vaccine counseling and administration, difficulty tracking patients to complete the vaccine series and relatively low uptake [90,91]. In addition, although all 50 states have laws allowing minors over age 13 to receive diagnosis and treatment of STIs without parental consent, many state laws do not explicitly cover prevention in a way that would allow extension to vaccination [92].

\section{Family planning clinics}

Reach: Over 3000 Title X and other family planning clinics provide care to the US adolescents and adults, largely targeting socioeconomically disadvantaged populations at high risk for contracting STIs [13]. Many of these clinics are in rural regions of the country, offering care to at-risk and underserved females and males who may not have access to routine primary care [13]. However, much as with OBGYN and STI clinics, many patients of family planning clinics are already sexually active, making the vaccine likely to be less effective in this population.

Acceptability: Currently, little evidence suggests parents and adolescents would seek out family planning clinics for HPV vaccine or other adolescent vaccines. Although recognized 
as a potential setting to expand adolescent immunization $[13,16]$, parents rate family planning clinics as one of the least desirable location for their adolescents to receive vaccinations as compared with several other sites such as school health and public health clinics [16]. Among adolescents themselves, only 35\% of 6th grade and 55\% of 11th grade students found vaccination in a family planning clinics acceptable, the lowest percentage among the surveyed alternative settings [93].

Feasibility: Many family planning clinics have experience providing immunizations related to STIs such as hepatitis B, providing a tested platform for HPV vaccination [94]. Implementing HPV vaccination services may be easier in family planning clinics than in STI clinics because of the emphasis on preventive care. To ensure series completion, the vaccine series could be timed with routine follow-up visits such as those related to contraception refills.

\section{Emergency departments}

Reach-Around 5\% of the US adolescents, most of whom are uninsured, use emergency departments as their only source of healthcare [17,95]. This figure may substantially decrease in years to come as more states expand their Medicaid services to the uninsured or underserved under the ACA.

Acceptability-Parents and adolescents generally find emergency departments to be an acceptable site to get vaccines [14,93], though these studies have not specifically addressed HPV vaccine. As preventive healthcare visits are inconsistent with the core practice model of providing acute care within emergency departments, emergency department staff typically refer patients to primary care providers or public health clinics for such services that may also have better access to vaccination histories [96].

Feasibility-Significant obstacles limit the ability of emergency departments to vaccinate adolescents, especially in the provision of serial vaccines such as HPV. While the most frequently delivered immunization in emergency departments is tetanus (93\%) [97] as emergency post-exposure prophylaxis for injuries, widespread provision of other vaccines within emergency departments may not be cost-effective since reimbursements may be lower than the administration costs or insurers may not cover the cost of care at all [13]. Some pilot programs have shown that creating an adolescent immunization platform in emergency departments is effective in capturing hard-to-reach adolescent populations, although such programs have had difficulty demonstrating sustainability [13]. Moreover, adolescents generally use emergency department services for acute care, making it difficult to ensure that patients who start the multi-dose HPV series complete it.

\section{Dental practices}

Reach-Around $80 \%$ of children and adolescents have had a dental visit within the past 12 months [98]. While racial and ethnic disparities in dental visits have been significant in the past, recent data show that such differences have been attenuated substantially [98]. The high access to adolescents may make dental practices worth considering as a setting for HPV vaccine provision. 
Acceptability-Though the link between HPV and oral cancer provides a rationale for involving dentists, parents' and adolescents' comfort with receiving HPV vaccination in a dental office is not known. Dentists and dental hygienists are becoming more aware of the link between HPV infection and oral cancer, but they have a wide variation in knowledge and attitudes surrounding HPV-associated oral cancer, HPV vaccine and the role of the provider in counseling and raising awareness about such topics [99].

Feasibility-With no precedent for vaccination or infrastructure to support it in terms of vaccine refrigeration, training to deliver doses and status as in-network providers, administration of HPV vaccine is not currently feasible in most dental offices. Although dentists are trained in medical procedures involving injections, they are not trained to administer vaccines or to monitor for related side effects. The rapidly increasing rate of oropharyngeal cancer may create new interest among dentists to expand beyond the current sporadically adopted practice of oral cancer screening. Additional research on revising dental practice and developing policies to help standardize these practices could help dentists play a more central role in HPV vaccination in the future.

\section{Expert commentary}

Dr Tom Frieden, the Director of the CDC, stated in 2013 that progress in HPV vaccination had stalled, a failure of providing the vaccine primarily through traditional primary care settings [100]. Reaching the nation's goal of widespread HPV vaccination will require interventions in traditional settings, but these interventions should be paired with efforts in alternative settings. Use of pharmacies and mass vaccination efforts in schools are among the most promising strategies because of their high reach, good acceptability and potential for feasible implementation. Other alternative settings in specialty practices (OBGYN, STI and family planning), emergency departments and dental offices were less promising.

A key challenge of alternative settings is creating demand for their vaccination services among parents and adolescents. To some extent, comfort with these alternative settings will increase over time as settings like pharmacies become more common locations for adult vaccination and other services. Promotion of vaccination services by settings like pharmacies will also help. Other settings like schools may benefit from evidence-based tools for boosting parent and adolescent interest in and consent to vaccination, especially in the absence of effective school entry requirements for HPV vaccination. Such requirements for Tdap and meningococcal vaccines have substantially increased adolescent vaccine coverage in the USA [101], but similar requirements for HPV vaccine have been unpopular [102,103] and not very effective [104]. No-cost, voluntary mass vaccination programs have been well received and as a result achieved high immunization rates in other countries [104]. State vaccination registries have reminder-recall functions that can increase immunization in adolescents [105]. Though primary care settings have largely failed to routinely use this effective intervention, adoption of such systems by alternative settings more accustomed to promotion could increase demand for HPV vaccine.

Alternative settings' ability to adequately document vaccine doses and to communicate with adolescents' primary care providers is of concern to parents and physicians $[44,56,74]$. 
Large chain pharmacies have record systems that are interoperable that allow any pharmacist within that network to access a patient's profile to verify the vaccination records. However, systems are not interoperable between different chains or independent pharmacies, and in many states, pharmacy practice laws do not require pharmacies to report administered vaccines back to patients' primary care providers or state immunization registries. Moreover, some pharmacists and providers in other alternative settings must rely on patient-reported vaccination history, because they do not yet have access to state vaccine registries or patients' medical records. Fostering coordinated care will require addressing the limited or lack of access to registries and medical records, along with establishing a standardized way for alternative settings to report vaccine doses to primary care providers. Mass vaccinations in schools that do not have electronic record keeping meet substantial challenges in reporting. By using state immunization registries and reporting vaccination to primary care providers, alternative HPV vaccine providers could address this barrier, as well as track vaccine delivery to ensure compliance.

The ability of alternative settings to bill private insurance will determine their long-term viability as HPV vaccine providers. Private insurance coverage for HPV vaccine currently varies widely by plan $[37,106]$. As the provisions of the ACA take effect, plans will provide first dollar coverage for ACIP-recommended vaccines, including HPV vaccine [106], but this will not establish alternative settings as in-network providers. Creating in-network status for pharmacists and school-located programs can go a long way in reducing the financial burden for both patients and providers, and will promote an environment that would allow adolescents and their parents the flexibility to choose where they receive their preventive care.

The federally funded VFC program that funds ACIP-recommended vaccines free of charge for eligible adolescents is central to vaccine provision in the USA, but many alternative settings may not yet have access to this program. Some states, such as Nevada, have readily available application forms and guidelines to help pharmacies enroll in and deliver vaccines through the VFC program [107], and the CDC offers continuing education to healthcare providers, including pharmacists, to learn about this program [108]. Pharmacies or other alternative settings choosing not to become VFC providers would substantially limit their reach.

While pharmacies and schools are the most promising alternative settings for HPV vaccination, other alternative settings may play a small role in providing the vaccine. Most OBGYN practices already provide HPV vaccine, but they miss the opportunity to vaccinate the majority of their patients, and their reach to adolescents is limited. Specialty practices like STI and family planning clinics, constrained by federal and state funding, may struggle to expand HPV immunization programs effectively despite their past experiences with vaccination services. Continued financial and legislative support for these clinics that deliver HPV vaccine may help provide a safety net for patients who are socioeconomically disadvantaged, but will never have as meaningful of a reach as primary care clinics, pharmacies or mass vaccination in schools. Additionally, family planning and STI clinics are not preferred vaccination sites among parents, making these clinics less promising alternative settings as compared with pharmacies and schools. Also, issues surrounding 
scope of practice in some of these alternative settings pose large barriers. Emergency departments and dental clinics are not presently viable as alternative settings for delivering HPV vaccine.

\section{Five-year view}

Lost opportunities to vaccinate this generation of adolescents will result in needless HPVrelated disease burden in the USA in the years to come. An estimated 53,000 cases of HPVrelated cancers could potentially be prevented in the future if $80 \%$ of today's US adolescents aged 11-12 completed the HPV series [28]. The lost opportunity will be even larger once the FDA approves a new nonavalent HPV vaccine that may prevent almost all cervical cancers and the majority of high-grade pre-cancers [109]. While improving HPV vaccine provision in primary care will be an important area of intervention, increasing use of alternative vaccination settings can help offset some of the burden on our nation's primary care providers, and provide more opportunities to vaccinate the US adolescents.

We can address some of the challenges to HPV vaccine delivery in alternative settings by using already identified best practices. Future research and program implementation with pharmacy provision of HPV vaccine should explore collaborative practice models with primary care providers. Collaborative practice agreements allow physicians to extend their capacity for patient care by having patients receive some preventive services such as HPV vaccination at a local pharmacy. One possibility to address physician organizations' concerns about pharmacists-provided HPV vaccination is for a primary care provider to administer the initial vaccine dose, and then allow community pharmacists to complete the series [48]. This referral method could ease the burden on parents for scheduling multiple additional primary care visits by providing better hours, more convenient locations and possibly lowering costs, while providing similar quality of care as primary care clinics.

Future iterations of mass vaccination programs in schools could take several approaches to be more successful. First, schools can form community partnerships with health departments, community clinics and pharmacies to furnish the vaccine to their students [77]. Strategies to accomplish this goal include harnessing the existing capacity of local health departments to organize large-scale health interventions [77]. Second, school-located programs should focus on adolescent vaccines, not just HPV vaccine, to minimize reactance [59]. Third, these programs should be hosted at the schools and not offsite to improve participation [76]. Fourth, best practices to improve consent procedures may increase participation among adolescents and their parents. For example, schools can: offer incentives to individual children or peer groups for returning forms that indicate whether parents give consent; design consent forms that are simple, using visual clues to identify where parents should sign and are printed in all relevant languages [110]; and use forms that allow parents the option to approve all vaccines, rather than each vaccine individually [111]. Importantly, schools offering vaccines have been more successful when providing vaccinerelated information through multiple mechanisms such as teacher-led student assemblies [112] and dedicated websites for vaccine information [67]. Finally, best practices and recommendations also exist for the physical layout of the vaccination setting [113] and the preparation and flow of students through the vaccine delivery area [112]. 
Among the many potential alternative settings, we believe that pharmacies and schools have the largest potential for increasing HPV vaccine coverage (Table 1). These settings offer high reach, convenience and a history of successful immunization in and outside of the USA. While many pharmacies and schools do not yet have the capacity to pursue strategies that we outline in our paper (e.g., expanding insurance coverage or collaborative practice models in pharmacies), such strategies provide future directions that researchers and policymakers can take to test the feasibility of providing HPV vaccine in these settings.

\section{References}

Papers of special note have been highlighted as:

- of interest

•• of considerable interest

1. Smith JS, Lindsay L, Hoots B, et al. Human papillomavirus type distribution in invasive cervical cancer and high-grade cervical lesions: a meta-analysis update. Int J Cancer. 2007; 121(3):621-32. [PubMed: 17405118]

2. Lowy DR, Schiller JT. Reducing HPV-associated cancer globally. Cancer Prev Res. 2012; 5(1):1823.

3. Newmann SJ, Garner EO. Social inequities along the cervical cancer continuum: a structured review. Cancer Causes Control. 2005; 16(1):63-70. [PubMed: 15750859]

4. Markowitz LE, Dunne E, Saraiya M, et al. Quadrivalent human papillomavirus vaccine. MMWR Morb Mortal Wkly Rep. 2007; 56(RR02):1-24. [PubMed: 17218934]

5. Dunne E, Markowitz L, Chesson H, et al. Recommendations on the use of quadrivalent human papillomavirus vaccine in males-Advisory Committee on Immunization Practices (ACIP), 2011. MMWR Morb Mortal Wkly Rep. 2011; 60(50):1705-8. [PubMed: 22189893]

6. Centers for Disease Control and Prevention (CDC). NIS-Teen Vaccination Coverage Table Data. 2013. 2012. Available from: http://www.cdc.gov/vaccines/stats-surv/nisteen/tables/12/ tab01_iap_2012.pdf

7. Tan W, Viera AJ, Rowe-West B, et al. The HPV vaccine: are dosing recommendations being followed? Vaccine. 2011; 29(14):2548-54. [PubMed: 21300098]

8. Strickland BB, Jones JR, Ghandour RM, et al. The medical home: health care access and impact for children and youth in the United States. Pediatrics. 2011; 127(4):604-11. [PubMed: 21402643]

9. Bodenheimer T, Pham HH. Primary care: current problems and proposed solutions. Health Aff. 2010; 29(5):799-805.

10. Elster A, Jarosik J, VanGeest J, Fleming M. Racial and ethnic disparities in health care for adolescents: a systematic review of the literature. Arch Pediatr Adolesc Med. 2003; 157(9):86774. [PubMed: 12963591]

11. Newacheck PW, Hung YY, Jane Park M, et al. Disparities in adolescent health and health care: does socioeconomic status matter? Health Serv Res. 2003; 38(5):1235-52. [PubMed: 14596388]

12. President's Cancer Panel. President's Cancer Panel Annual Report 2012-2013. National Institutes of Health; 2013. Accelerating HPV vaccine uptake: urgency for action to prevent cancer.

13••. Schaffer SJ, Fontanesi J, Rickert D, et al. How effectively can health care settings beyond the traditional medical home provide vaccines to adolescents? Pediatrics. 2008; 121(Suppl 1):S3545. In 2008, a CDC working group of vaccination experts reported an evaluation of several vaccination settings outside of the medical home. They established that the discussed settings were safe and appropriate to deliver adolescent vaccines. [PubMed: 18174319]

14. Rand CM, Shone LP, Albertin C, et al. National health care visit patterns of adolescents: implications for delivery of new adolescent vaccines. Arch Pediatr Adolesc Med. 2007; 161(3): 252-9. [PubMed: 17339506]

Expert Rev Vaccines. Author manuscript; available in PMC 2015 February 01. 
15. Marcell AV, Klein JD, Fischer I, et al. Male adolescent use of health care services: where are the boys? J Adolesc Health. 2002; 30(1):35-43. [PubMed: 11755799]

16•. Clevenger L, Pyrzanowski J, Curtis CR, et al. Parents' acceptance of adolescent immunizations outside of the traditional medical home. J Adolesc Health. 2011; 49(2):133-40. Parents found that vaccination in public health clinics, schools, OBGYN clinics and emergency departments as acceptable alternatives to vaccination in primary care clinics. [PubMed: 21783044]

17. Klein JD, McNulty M, Flatau CN. Adolescents' access to care: teenagers' self-reported use of services and perceived access to confidential care. Arch Pediatr Adolesc Med. 1998; 152(7):676. [PubMed: 9667540]

18•. Rand CM, Szilagyi PG, Albertin C, Auinger P. Additional health care visits needed among adolescents for human papillomavirus vaccine delivery within medical homes: a national study. Pediatrics. 2007; 120(3):461-6. Most adolescents would require two to three additional primary care visits to complete the human papillomavirus (HPV) vaccine series, and additional efforts by primary care providers, and the use of alternative settings must be made to ensure series completion. [PubMed: 17766516]

19. Gerend MA, Weibley E, Bland H. Parental response to human papillomavirus vaccine availability: uptake and intentions. J Adolesc Health. 2009; 45(5):528-31. [PubMed: 19837361]

20. Reiter PL, Brewer NT, Gottlieb SL, et al. Parents' health beliefs and HPV vaccination of their adolescent daughters. Soc Sci Med. 2009; 69(3):475-80. [PubMed: 19540642]

21. Allen JD, Othus MK, Shelton RC, et al. Parental decision making about the HPV vaccine. Cancer Epidemiol Biomarkers Prev. 2010; 19(9):2187-98. [PubMed: 20826829]

22 . Brewer NT, Gottlieb SL, Reiter PL, et al. Longitudinal predictors of human papillomavirus vaccine initiation among adolescent girls in a high-risk geographic area. Sex Transm Dis. 2011; 38(3):197-204. 63\% of unvaccinated adolescent girls who had been to the doctor in the previous year had not received a recommendation for HPV vaccine; $62 \%$ of parents who intended to vaccinate were not able to follow through on these good intentions. [PubMed: 20838362]

23. Rosenthal SL, Weiss TW, Zimet GD, et al. Predictors of HPV vaccine uptake among women aged 19-26: importance of a physician's recommendation. Vaccine. 2011; 29(5):890-5. [PubMed: 20056186]

24. Lau M, Lin H, Flores G. Factors associated with human papillomavirus vaccine-series initiation and healthcare provider recommendation in US adolescent females. 2007 National Survey of Children's Health Vaccine. 2012; 30(20):3112-18.

25. Dorell C, Stokley S, Yankey D, et al. National and state vaccination coverage among adolescents aged 13-17 Years-United States 2011. MMWR Morb Mortal Wkly Rep. 2012; 61:671-7. [PubMed: 22932301]

26. Hughes CC, Jones AL, Feemster KA, Fiks AG. HPV vaccine decision making in pediatric primary care: a semi-structured interview study. BMC Pediatr. 2011; 11:74. [PubMed: 21878128]

27. Hamlish T, Clarke L, Alexander KA. Barriers to HPV immunization for African American adolescent females. Vaccine. 2012; 30(45):6472-6. [PubMed: 22910288]

28••. Stokley S, Curtis CR, Jeyarajah J, et al. Human papillomavirus vaccination coverage among adolescent girls, 2007-2012, and postlicensure vaccine safety monitoring, 2006-2013 - United States. MMWR Morb Mortal Wkly Rep. 2013; 62(29):591-5. The CDC reports that HPV vaccination among adolescent girls did not increase from 2011 to 2012. [PubMed: 23884346]

29. Bynum SA, Staras SA, Malo TL, et al. Factors associated with Medicaid providers' recommendation of the HPV vaccine to low-income adolescent girls. J Adolesc Health. 2013 In press.

30. Dempsey AF, Davis MM. Overcoming barriers to adherence to HPV vaccination recommendations. Am J Manag Care. 2006; 12(17 Suppl):S484-91. [PubMed: 17203992]

31. Vadaparampil ST, Kahn JA, Salmon D, et al. Missed clinical opportunities: provider recommendations for HPV vaccination for 11-12 year old girls are limited. Vaccine. 2011; 29(47): 8634-41. [PubMed: 21924315]

32. Szilagyi PG, Rand CM, McLaurin J, et al. Delivering adolescent vaccinations in the medical home: a new era? Pediatrics. 2008; 121(Suppl 1):S15-24. [PubMed: 18174317] 
33. Gilkey MB, Moss JL, McRee AL, Brewer NT. Do correlates of HPV vaccine initiation differ between adolescent boys and girls? Vaccine. 2012; 30(41):5928-34. [PubMed: 22841973]

34. Campos-Outcalt D, Jeffcott-Pera M, Carter-Smith P, et al. Vaccines provided by family physicians. Ann Fam Med. 2010; 8(6):507-10. [PubMed: 21060120]

35. Reiter PL, McRee A, Pepper JK, et al. Improving human papillomavirus vaccine delivery: a national study of parents and their adolescent sons. J Adolesc Health. 2012; 51(1):32-7. [PubMed: 22727074]

36. Katz ML, Reiter PL, Kluhsman BC, et al. Human papillomavirus (HPV) vaccine availability, recommendations, cost, and policies among health departments in seven Appalachian states. Vaccine. 2009; 27(24):3195-200. [PubMed: 19446191]

37. Centers for Disease Control and Prevention (CDC). HPV Vaccine - Questions \& Answers. 2012. Available from http://www.cdc.gov/vaccines/vpd-vac/hpv/vac-faqs.htm

38. The American Pharmacists Association. Every month should be immunization month...Until everyone is vaccinated. Adapted from a document presented to HHS Secretary Shalala and the National Vaccination Program Office; Washington, DC, USA. 1994;

39. Knapp KK. Building a pharmacy work force mosaic: new studies help to fill in the gaps. J Am Pharm Assoc. 2000; 40(1):13-14.

40. Bureau of Labor Statistics, US Department of Labor. Occupational outlook handbook 2012-2013: pharmacists. 2012. Available from: http://www.bls.gov/oes/current/oes291051.htm

41. Pilisuk T, Goad J, Backer H. Vaccination delivery by chain pharmacies in California: results of a 2007 survey. J Am Pharm Assoc. 2010; 50(2):134-9.

42. Manolakis PG, Skelton JB. Pharmacists' contributions to primary care in the United States collaborating to address unmet patient care needs: the emerging role for pharmacists to address the shortage of primary care providers. Am J Pharm Educ. 2010; 74(10):1. [PubMed: 20221352]

43. Gallup. Honesty/ethics in professions. 2012. Available from: http://www.gallup.com/poll/1654/ honesty-ethics-professions.aspx

44. McRee A, Reiter PL, Pepper JK, Brewer NT. Correlates of comfort with alternative settings for HPV vaccine delivery. Hum Vaccin Immunother. 2013; 9(2):42-9.

45. Rosenbluth SA, Madhavan SS, Borker RD, Maine LL. Pharmacy immunization partnerships: a rural model. J Am Pharm Assoc. 2001; 41(1):100-7.

46. Ndiaye SM, Madhavan S, Washington ML, et al. The use of pharmacy immunization services in rural communities. Public Health. 2003; 117(2):88-97. [PubMed: 12802974]

47. Fontanesi J, Hirsch J, Lorentz S, et al. Comparison of pharmacists and primary care providers as immunizers. Am J Pharm Benefits. 2009; 1(3):50-7.

48. McIntosh J, Sturpe DA, Khanna N. Human papillomavirus vaccine and cervical cancer prevention: practice and policy implications for pharmacists. J Am Pharm Assoc. 2008; 48(1):e1-17.

49. Romanelli F, Freeman T. Immunization training: right or privilege? Am J Pharm Educ. 2012; 76(4):1. [PubMed: 22412200]

50. Brewer NT, Chung JK, Baker HM, Smith JS. Pharmacist immunization authority for HPV, Tdap, and meningococcal vaccines. Under review. 2013

51. Committee on Practice and Ambulatory Medicine and Council on Community Pediatrics. Increasing immunization coverage. Pediatrics. 2010; 125(6):1295-304. [PubMed: 20513736]

52. Lofink, H.; Kuebler, J.; Juszczak, L., et al. 2010-2011 School-Based Health Alliance Census Report. 2013. Available from: http://www.sbh4all.org/atf/cf/\%7BB241D183DA6F-443F-9588-3230D027D8DB\%7D/2010-11\%20Census\%20Report\%20Final.pdf

53. Brener ND, Wheeler L, Wolfe LC, et al. Health services: results from the school health policies and programs study 2006. J Sch Health. 2007; 77(8):464-85. [PubMed: 17908103]

54. School Based Health Alliance (SBHA). SBHC capital grant. 2012. Available from: http:// www.sbh4all.org/site/c.ckLQKbOVLkK6E/b.7697107/apps/s/content.asp?ct=11666829

55. Federico SG, Abrams L, Everhart RM, et al. Addressing adolescent immunization disparities: a retrospective analysis of school-based health center immunization delivery. Am J Public Health. 2010; 100(9):1630. [PubMed: 20634466] 
56••. Lindley MC, Boyer-Chu L, Fishbein DB, et al. The role of schools in strengthening delivery of new adolescent vaccinations. Pediatrics. 2008; 121(Suppl 1):S46-54. Position paper providing physician support for schools as a vaccination setting, and outlining challenges and opportunities to expand school vaccination services. [PubMed: 18174320]

57. Strozer, J.; Juszczak, L.; Ammerman, A. 2007-2008 National School-based health care census. National Assembly on School-Based Health Care; Washington, DC: 2010.

58. Daley MF, Curtis CR, Pyrzanowski J, et al. Adolescent immunization delivery in school-based health centers: a national survey. J Adolesc Health. 2009; 45(5):445-2. [PubMed: 19837350]

59. Moss JL, Leighton A, O’Malley B, et al. Opportunities for increasing HPV vaccine provision in school health centers. Submitted.

60. Fiks AG, Grundmeier RW, Biggs LM, et al. Impact of clinical alerts within an electronic health record on routine childhood immunization in an urban pediatric population. Pediatrics. 2007; 120(4):707-14. [PubMed: 17908756]

61. Hayes KA, Entzel P, Berger W, et al. Early lessons learned from extramural school programs that offer HPV vaccine. J Sch Health. 2013; 83(2):119-26. [PubMed: 23331272]

62. US Census Bureau. School enrollment. 2013. Available from: http://www.census.gov/hhes/school/ data/cps/2012/tables.html

63. Stretch R, Roberts S, McCann R, et al. Parental attitudes and information needs in an adolescent HPV vaccination programme. Br J Cancer. 2008; 99(11):1908-11. [PubMed: 18985038]

64. Reeve C, De La Rue S, Pashen D, et al. School-based vaccinations delivered by general practice in rural north Queensland: an evaluation of a new human papilloma virus vaccination program. Commun Dis Intell. 2008; 32(1):94.

65. Watson M, Shaw D, Molchanoff L, McInnes C. Challenges, lessons learned and results following the implementation of a human papilloma virus school vaccination program in South Australia. Aust NZ J Public Health. 2009; 33(4):365-70.

66. Brotherton JM, Fairley CK, Garland SM, et al. Closing editorial: processes, opportunities and challenges after introduction of human papillomavirus vaccine. Sex Health. 2010; 7(3):397-8. [PubMed: 21058499]

67. Ogilvie G, Anderson M, Marra F, et al. A population-based evaluation of a publicly funded, school-based HPV vaccine program in British Columbia, Canada: parental factors associated with HPV vaccine receipt. PLoS Med. 2010; 7(5):e1000270. [PubMed: 20454567]

68. Musto R, Siever JE, Johnston JC, et al. Social equity in Human Papillomavirus vaccination: a natural experiment in Calgary Canada. BMC Public Health. 2013; 13(1):640. [PubMed: 23837819]

69. Hollinger F. Comprehensive control (or elimination) of hepatitis B virus transmission in the United States. Gut. 1996; 38(Suppl 2):S24-30. [PubMed: 8786049]

70. King JC, Stoddard JJ, Gaglani MJ, et al. Effectiveness of school-based influenza vaccination. N Engl J Med. 2006; 355(24):2523-32. [PubMed: 17167135]

71. Effler PV, Chu C, He H, et al. Statewide school-located influenza vaccination program for children 5-13 years of age, Hawaii, USA. Emerg Infect Dis. 2010; 16(2):244-50. [PubMed: 20113554]

72. Vanderpool RC, Cohen EL, Crosby RA, et al. "1-2-3 Pap” Intervention improves HPV vaccine series completion among Appalachian women. J Commun. 2013; 63(1):95-115.

73. Cooper Robbins SC, Ward K, Skinner SR. School-based vaccination: a systematic review of process evaluations. Vaccine. 2011; 29(52):9588-99. [PubMed: 22033031]

74. Kadis JA, McRee A, Gottlieb SL, et al. Mothers' support for voluntary provision of HPV vaccine in schools. Vaccine. 2011; 29(14):2542-7. [PubMed: 21300097]

75. Kelminson K, Saville A, Seewald L, et al. Parental views of school-located delivery of adolescent vaccines. J Adolesc Health. 2012; 51(2):190-6. [PubMed: 22824451]

76. Stubbs BW, Panozzo CA, Moss JL, et al. Evaluation of an intervention providing HPV vaccine in schools. Am J Health Behav. 2014; 38(1):92-102. [PubMed: 24034684]

77. Lott J, Johnson J. Promising practices for school-located vaccination clinics—part i: preparation. Pediatrics. 2012; 129(Suppl 2):S75-80. [PubMed: 22383485]

78. Nelson R. School nurses are needed more than ever. Am J Nurs. 2009; 109(12):25-7. 
79. Hoover KW, Tao G, Berman S, Kent CK. Utilization of health services in physician offices and outpatient clinics by adolescents and young women in the United States: implications for improving access to reproductive health services. J Adolesc Health. 2010; 46(4):324-30. [PubMed: 20307820]

80. Carlos RC, Dempsey AF, Patel DA, Dalton VK. Cervical cancer prevention through human papillomavirus vaccination: using the "teachable moment" for educational interventions. Obstet Gynecol. 2010; 115(4):834. [PubMed: 20308846]

81. Salz T, Gottlieb SL, Smith JS, Brewer NT. The association between cervical abnormalities and attitudes toward cervical cancer prevention. J Womens Health. 2010; 19(11):2011-16.

82. Vitek WS, Akers A, Meyn LA, et al. Vaccine eligibility and acceptance among ambulatory obstetric and gynecologic patients. Vaccine. 2011; 29(11):2024-8. [PubMed: 21272604]

83. Perkins RB, Anderson BL, Gorin SS, Schulkin JA. Challenges in cervical cancer prevention: a survey of U.S. obstetrician-gynecologists. Am J Prev Med. 2013; 45(2):175-81. [PubMed: 23867024]

84. American College of Obstetricians and Gynecologists. Human papillomavirus vaccination. committee opinion No 467. Obstet Gynecol. 2010; 116(3):800-3. [PubMed: 20733476]

85. Leddy MA, Anderson BL, Power ML, et al. Changes in and current status of obstetriciangynecologists' knowledge, attitudes, and practice regarding immunization. Obstet Gynecol Surv. 2009; 64(12):823-9. [PubMed: 19939296]

86. Schrag SJ, Fiore AE, Gonik B, et al. Vaccination and perinatal infection prevention practices among obstetrician-gynecologists. Obstet Gynecol. 2003; 101(4):704-10. [PubMed: 12681874]

87. Power ML, Leddy MA, Anderson BL, et al. Obstetrician-Gynecologists' practices and perceived knowledge regarding immunization. Am J Prev Med. 2009; 37(3):231-4. [PubMed: 19596538]

88. Celum CL, Bolan G, Krone M, et al. Patients attending STD clinics in an evolving health care environment: demographics, insurance coverage, preferences for STD services, and STD morbidity. Sex Transm Dis. 1997; 24(10):599-605. [PubMed: 9383850]

89. Meites E, Llata E, Hariri S, et al. HPV vaccine implementation in STD clinics -STD surveillance network. Sex Transm Dis. 2012; 39(1):32-4. [PubMed: 22183843]

90. Cummingswilson B, Moyer L, Schmid G, et al. Hepatitis B vaccination in sexually transmitted disease (STD) clinics: a survey of STD programs. Sex Transm Dis. 2001; 28(3):148-52. [PubMed: 11289196]

91. Handsfield HH. Hepatitis A and B immunization in persons being evaluated for sexually transmitted diseases. Am J Med. 2005; 118(10):69-74.

92. English A, Shaw FE, McCauley MM, Fishbein DB. Legal basis of consent for health care and vaccination for adolescents. Pediatrics. 2008; 121(Suppl 1):S85-7. [PubMed: 18174325]

93. Pyrzanowski J, Curtis CR, Crane LA, et al. Adolescents' perspectives on vaccination outside the traditional medical home a survey of urban middle and high school students. Clin Pediatr. 2013; 52(4):329-37.

94. Gilbert LK, Bulger J, Scanlon K, et al. Integrating hepatitis B prevention into sexually transmitted disease services: US sexually transmitted disease program and clinic trends-1997 and 2001. Sex Transm Dis. 2005; 32(6):346-50. [PubMed: 15912080]

95. Richardson LD, Asplin BR, Lowe RA. Emergency department crowding as a health policy issue: past development, future directions. Ann Emerg Med. 2002; 40(4):388-93. [PubMed: 12239493]

96. American College of Emergency Physicians, Pediatric Committee. . Immunization of adults and children in the emergency department. Ann Emerg Med. 2008; 51(5):695. [PubMed: 18436062]

97. Pallin DJ, Muennig PA, Emond JA, et al. Vaccination practices in U.S emergency departments, 1992-2000. Vaccine. 2005; 23(8):1048-52. [PubMed: 15620478]

98. Isong IA, Soobader M, Fisher-Owens SA, et al. Racial disparity trends in children's dental visits: US national health interview survey, 1964-2010. Pediatrics. 2012; 130(2):306-14. [PubMed: 22753556]

99. Daley E, DeBate R, Dodd V, et al. Exploring awareness, attitudes, and perceived role among oral health providers regarding HPV-related oral cancers. J Public Health Dent. 2011; 71(2):136-42. [PubMed: 21774137] 
100. Centers for Disease Control and Prevention (CDC). HPV vaccine: safe, effective, and grossly underutilized. 2013. Available from: http://www.cdc.gov/media/releases/2013/p0725-HPVvaccine.html

101. Bugenske E, Stokley S, Kennedy A, Dorell C. Middle school vaccination requirements and adolescent vaccination coverage. Pediatrics. 2012; 129(6):1056-63. [PubMed: 22566425]

102. Smith JS, Brewer NT, Chang Y, et al. Acceptability of school requirements for human papillomavirus vaccine. Hum Vaccin. 2011; 7(9):952-7. [PubMed: 22024912]

103. Robitz R, Gottlieb SL, De Rosa CJ, et al. Parent attitudes about school requirements for human papillomavirus vaccine in high-risk communities of Los Angeles, California. Cancer Epidemiol Biomarkers Prev. 2011; 20(7):1421-9. [PubMed: 21551243]

104. Gilkey MB, Brewer NT. Mandatory HPV vaccination. JAMA. 2012; 307(3):252-3. [PubMed: 22253386]

105. Suh CA, Saville A, Daley MF, et al. Effectiveness and net cost of reminder/recall for adolescent immunizations. Pediatrics. 2012; 129(6):e1437-45. [PubMed: 22566415]

106. Singer DC, Davis MM, Gebremariam A, Clark SJ. Underinsurance for recently recommended vaccines in private health plans. J Community Health. 2012; 37(6):1164-7. [PubMed: 22562599]

107. Nevada Division of Public and Behavioral Health. Vaccines for Children Program. 2013. Available from: http://www.health.nv.gov/Vaccine_VFCProgram.htm

108. Centers for Disease Control and Prevention (CDC). WB2219-Immunization: you Call the ShotsModule Sixteen-Vaccines for Children Program. 2013. Available from: http:// www2a.cdc.gov/nip/isd/ycts/mod1/courses/vfc/ce.asp

109. Sharp, Merck; Corp, Dohme. Merck’s Investigational 9-valent HPV Vaccine, V503, Prevented 97 Percent of Cervical, Vaginal and Vulvar Pre-cancers Caused by Five Additional HPV Types, in Phase III Study. 2013. Available from: http://www.mercknewsroom.com/news-release/researchand-development-news/mercks-investigational-9-valent-hpv-vaccine-v503-prevente

110. Cawley J, Hull HF, Rousculp MD. Strategies for implementing school-located influenza vaccination of children: a systematic literature review. J Sch Health. 2010; 80(4):167-75. [PubMed: 20433642]

111. Golden SD, Moracco KE, Leighton A, et al. Process evaluation of an intervention to increase provision of adolescent vaccines at school health centers. J Sch Health. Under review.

112. Robbins SCC, Bernard D, McCaffery K, Skinner SR. 'It's a logistical nightmare!' Recommendations for optimising human papillomavirus school-based vaccination experience. Sex Health. 2010; 7(3):271-8. [PubMed: 20719214]

113. Kent H, Heffernan ME, Silvers J, et al. Role of the nurse immunizer in implementing and maintaining the national human papillomavirus 'cervical cancer' vaccine rollout through a school-based program in Victoria. Sex Health. 2010; 7(3):391-3. [PubMed: 20719232] 


\section{Key issues}

- Human papillomavirus (HPV) vaccine coverage among adolescents remains low as initial increases in coverage have stagnated, despite national recommendations.

- Causes of low HPV vaccination include missed opportunities in traditional primary care settings to vaccinate and inadequate primary care provider recommendations to adolescents and their parents.

- Pharmacies and voluntary mass vaccination programs in schools are already effectively providing vaccines in the USA.

- The most promising alternative settings are pharmacies and voluntary mass vaccination programs in schools, in part because they have the greatest potential reach to adolescents aged 11-12.

- Specialty clinics are less promising alternative sites as they have more limited access to adolescents, have low acceptability as providers of HPV vaccine or face substantial challenges to feasibility.

- Emergency departments and dental practices do not prioritize HPV vaccination because of scope of practice and may not play a large role in HPV vaccine provision without revision to practice.

- Expanding access to immunization registries, developing reimbursement mechanisms and maximizing care coordination will help make alternative settings more acceptable and sustainable for HPV vaccination. 


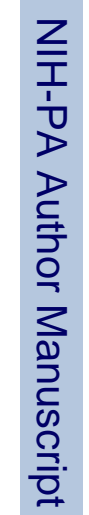

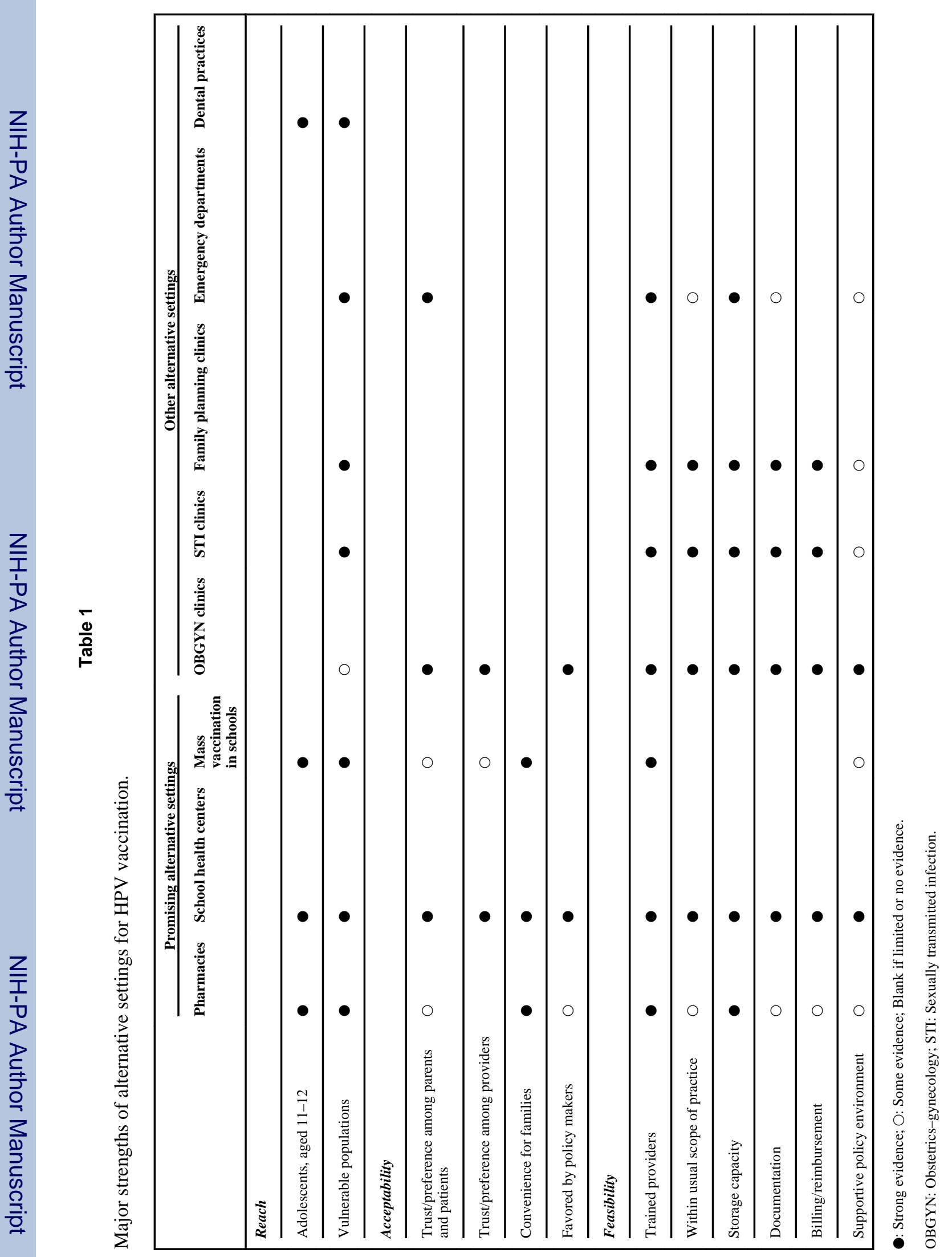

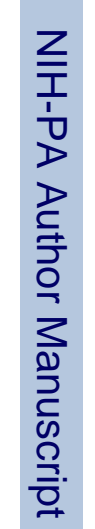

Expert Rev Vaccines. Author manuscript; available in PMC 2015 February 01. 